\title{
Una Noción de Evaluación: La Evaluación Formativa en el Marco de las Concepciones de Finlandia y la Ciudad de Buenos Aires
}

\author{
An Assessment Notion: Formative Assessment in the Frame of \\ the Conceptions in Finland and the City of Buenos Aires
}

Celina Cardoner Zunino*

Universidad de San Andrés

\begin{abstract}
El término evaluación ha sido históricamente una palabra que se utiliza frecuentemente en la práctica docente, con distintos sentidos. Existen diferencias respecto del modo de conceptualizarla que hacen que la evaluación de los aprendizajes sea una temática de difícil resolución. Este informe de investigación se propone identificar los argumentos generados en torno a la cultura evaluativa de la ciudad de Buenos Aires, comparándolos con las tendencias de evaluación que existen en Finlandia. A su vez, luego de afirmar que la evaluación formativa es indispensable para la mejora en la calidad de los aprendizajes, se identificará la presencia o ausencia de argumentos que se inscriban o no en el paradigma de la evaluación formativa. Por ello, trabajando en torno a los propósitos de evaluación que se presentan en los Diseños Curriculares vigentes de escuela media -el National Core Curriculum for Upper Secondary Schools y el Diseño Curricular de la Nueva Escuela Secundaria- se identificarán las rupturas, los vacíos y concepciones respecto de la evaluación que se presentan formalmente para evaluar los aprendizajes. Los resultados demuestran que ambos documentos incorporan la evaluación formativa como herramienta para conocer y trabajar en el aprendizaje del alumno pero demuestran diferencias significativas en la concepción de sujeto que hay por detrás de esa pedagogía. Las autoridades educativas de ambas regiones exponen el deseo de atender personalmente a los alumnos dándoles feedback formativo pero, sin embargo, le exigen distintas habilidades al futuro egresado.
\end{abstract}

Palabras clave: Aprendizaje significativo, Retroalimentación, Evaluación formativa, Aprendizaje.

The term evaluation has historically been a word that is often used in teaching, with different meanings. There are differences in how to conceptualize that make learning assessment a topic difficult to resolve. This research report aims to identify the arguments generated around the evaluation culture of the city of Buenos Aires, comparing assessment trends that exist in Finland. In turn, after saying that formative assessment is essential for improving the quality of learning, we will identify the presence or absence of arguments that sign in or not in the paradigm of formative assessment. Therefore, working around evaluation purposes served by existing middle school curricular designs -the National Core Curriculum for Upper Secondary Schools and Curriculum Design of New School Secondary- we will identify breaks, gaps and conceptions regarding the assessment to be formally presented to assess learning on the middle level. Through the reading of documents, the ideas of evaluation and speeches about the subject role will make the analysis more profound and complex. The results show that both documents incorporate formative assessment as a tool to learn and work in student learning but show significant differences in the subject role that is put into plat behind this pedagogy. Education authorities in both regions exhibit a desire to personally attend training students giving feedback but, however, they require different skills to future graduates.

Keywords: Student achievement, Formative assessment, Feedback, Learning.

*Contacto: celinacardoner@gmail.com

issn: 1989-0397

www.rinace.net/riee/

https://revistas.uam.es/riee
Recibido:

7 de noviembre de 2015

$1^{\text {a }}$ Evaluación: 20 de enero de 2016

Aceptado: 16 de febrero de 2016 


\section{Introducción}

Desde que se creó el Programa Internacional para la Evaluación de Estudiantes (PISA), Argentina ha obtenido resultados que lo ubicaron entre los últimos puestos mientras que Finlandia siempre ha estado entre los primeros. Por lo tanto, el estado de la evaluación en la actualidad argentina introduce un marco complejo en el campo: la política educativa no se centra completamente en el alumno ni en las capacidades que los chicos deberían desarrollar en la escuela (habilidades intrapersonales, interpersonales y cognitivas). Desde el Ministerio de Educación, no sólo de la Ciudad Autónoma de Buenos Aires (CABA) sino también de Finlandia, se publicaron informes que describen la concepción de evaluación, la forma en la que se deberían evaluar los aprendizajes de los alumnos en la escuela media y las habilidades que se esperan de los alumnos egresados. Estos documentos son la puerta de entrada para hacer una primera aproximación a lo que se concibe como evaluación en las diferentes regiones. Ahora bien, partiendo del supuesto de que se plantean distintas estrategias, significados y silencios en torno a la evaluación de aprendizajes en las distintas regiones escogidas, este artículo contribuye en:

$\checkmark$ La exploración de las diversas concepciones paradigmáticas de evaluación de los aprendizajes que predominan en las prácticas educacionales de Buenos Aires y Finlandia.

$\checkmark$ La identificación de los modos y configuraciones que contribuyen y se inscriben en la noción de evaluación formativa en los distintos Diseños Curriculares de las regiones seleccionadas y el efecto que tienen en la constitución de un futuro egresado.

¿Cómo tiene que ser la evaluación de los aprendizajes para que el estado de esta cuestión no permanezca en decadencia? La respuesta se encuentra en los postulados de Scriven (1967), quien hace cincuenta años introdujo el término evaluación formativa. En los sistemas escolares actuales este tipo de evaluación debería ser de especial relevancia como dispositivo de pedagogía diferenciada. Se percibe una necesidad de individualizar el contenido, el ritmo y las modalidades de aprendizaje en función de objetivos definidos con claridad.

Por esto, la evaluación formativa es un quiebre, una ruptura al proponer dejar a un lado el grado de regulación grupal y trasladarlo a la individualización; busca una intervención acorde al proceso individual de cada alumno. De esta manera, el docente se enfrenta a un cambio abrupto en las dinámicas escolares en relación a la enseñanza, la administración de horarios, la organización de la clase, entre otras cosas.

Por su parte, la concepción cognitiva postula que el aprendizaje debe ser significativo y, para ello, el alumno debe interactuar con su entorno de manera tal que construya sus representaciones personales y debe realizar juicios de valor que le permitan tomar decisiones en base a ciertos parámetros de referencia. Es decir, la evaluación debe partir de los conocimientos y vivencias previas del sujeto para luego integrar el nuevo conocimiento y, de esa manera, generar aprendizaje significativo.

Es indispensable que en el proceso de evaluación se pongan en juego las capacidades del alumno, su comprensión, su análisis, sus actitudes, intereses y habilidades, para poder perfeccionar -a través de la retroalimentación- la acción educativa y el desarrollo integral del educando. Si la evaluación es formativa, entonces el aprendizaje será significativo. 
$\mathrm{Al}$ analizar los distintos documentos provenientes de las dos regiones, el National Core Curriculum for Upper Secondary Schools y el Diseño Curricular de la Nueva Escuela Secundaria (DCNES), se identifican las distintas propuestas institucionales que desarrolló cada gobierno para su sistema de evaluación y se ponen en juego las diferentes estrategias, tendencias, aportes y objetivos en relación a la evaluación y a lo que se espera del alumno.

Así se establece -por ejemplo- en el DCNES, que se espera que "la evaluación tenga como foco principal el logro de la autonomía personal y académica de los estudiantes, pudiendo desarrollarse mediante procesos de evaluación, autoevaluación y coevaluación” (Ministerio de Educación de la Ciudad de Buenos Aires, 2013, p. 145).

Por su parte, el National Core Curriculum for Upper Secondary Schools publicado por el Finnish National Board of Education plantea que la evaluación de los alumnos tiene como objetivo orientar y fomentar el aprendizaje y desarrollo de los estudiantes de diversas formas. El papel de la evaluación del aprendizaje de los estudiantes es proporcionarles información sobre sus avances y resultados de aprendizaje durante y tras la finalización de los estudios de enseñanza secundaria (Finnish National Board of Education, 2003).

A pesar de ser Finlandia parte de la Unión Europea y una región económicamente más activa que la Ciudad de Buenos Aires, forma parte de este análisis comparativo porque es un modelo de referencia dentro del mundo desarrollado. Es de utilidad conocer tanto las similitudes como las diferencias que rigen en la formalidad de los documentos para tener una mirada global de los distintos aterrizajes que tiene el concepto de evaluación en las regiones alrededor del mundo, especialmente de la región con mayor éxito educativo en los últimos años y en la región más representativa de la Argentina.

\section{Fundamentación de los documentos a analizar}

Los documentos a ser analizados son los diseños curriculares vigentes de escuela media de dos regiones diferentes. Por un lado, el National Core Curriculum for Upper Secondary Schools publicado por el Finnish National Board of Education en el año 2003 (está en proceso el nuevo diseño curricular que se va a lanzar en 2016); por otro lado, el Diseño Curricular de la Nueva Escuela Secundaria de la Ciudad de Buenos Aires publicado en el año 2013.

Se han elegido estos documentos por sobre otros porque, además de ser de circulación pública, son las propuestas formales que se presentan desde los ministerios y, por lo tanto, las propuestas que todas las instituciones deberían adquirir y poner en juego.

Ambos documentos presentan las dinámicas y lógicas institucionales que se esconden por detrás de los sistemas educativos y brindan una descripción exhaustiva de los contenidos a enseñar y las formas en las cuáles se distribuirán los contenidos a lo largo de la escolaridad secundaria. En tal sentido, estos documentos brindan herramientas para que las distintas instituciones planteen recorridos en los que se pongan en juego variadas formas de construcción, apropiación y reconstrucción de saberes para los estudiantes de nivel secundario. Además, proponen un marco común que permite reconocer y hacer lugar a la diversidad de trayectorias escolares, intereses y puntos de partida de los adolescentes.

Como fue planteado anteriormente, si bien Finlandia es un país que se encuentra en una región más desarrollada económicamente y posee diferencias culturales significativas, 
es relevante hacer una comparación debido al impacto que tiene este sistema educativo: recibe regularmente las mejores calificaciones a escala mundial y está reconocido como un modelo de referencia dentro del mundo desarrollado. Los resultados obtenidos periódicamente en el Informe PISA, así como el elevado índice de educación, respaldan al modelo igualitario y gratuito de educación en Finlandia.

Es habitual encontrar noticias en los diarios, publicaciones en Facebook o charlas TED que mencionen lo exitoso y prestigioso que es estudiar en Finlandia. Este país tiene el mejor sistema educativo del mundo por el valor que se le da a la profesión docente, por el método educativo que tiene, por cómo son los centros educativos, por la cultura y la política educativa. Por ese motivo, y también teniendo en cuenta los últimos resultados de las pruebas PISA, resulta eficiente e importante reconocer cómo circula el discurso de la evaluación formativa alrededor del mundo y cuál es el impacto que tiene en la mejora de la calidad de los aprendizajes. Se ha optado por el Diseño Curricular de la CABA por la relevancia e impacto que tiene en la matrícula escolar, siendo una de las ciudades del país con mayor número de escuelas públicas.

Sabiendo que el tema a desarrollar es la evaluación de los aprendizajes, hay ciertas secciones que serán de especial relevancia por sobre las demás presentadas en los distintos documentos. En el caso del DCNES, el capítulo se titula: "La evaluación en la NES” mientras que en el diseño curricular finlandés se presenta bajo el nombre de: "Assessment of student learning". Estas secciones exponen no sólo una concepción de evaluación particular sino también explican las herramientas puntuales a utilizar en el campo de la evaluación de los aprendizajes. Son estas las secciones que nos permitirán identificar la ausencia o presencia de argumentos sobre evaluación formativa.

De todas maneras, para conocer el perfil del egresado que se espera y relacionarlo con los propósitos de evaluación que promueve cada región, se tomarán los argumentos planteados en otras secciones de los documentos: las habilidades del educando, los objetivos de la escuela media, los contenidos y la cultura de enseñanza.

\section{Metodología}

En función de la naturaleza de los objetivos de la investigación y sabiendo que se intenta explorar el tipo de discurso que hay en torno a la evaluación y al perfil del egresado que se espera por parte de la CABA y Finlandia, la estrategia metodológica del estudio se inscribirá en una perspectiva cualitativa. Un estudio de esta índole nos permite recoger los discursos completos para proceder a la interpretación de los mismos en relación a la evaluación formativa, analizando las relaciones de significado que se producen con otras nociones.

Es decir, a través de un análisis documental de los diseños curriculares seleccionados nuestras fuentes de datos-, se pondrán en juego, en primer lugar, los argumentos de Cowen (2000) para poder hacer una lectura global de la cuestión. Esta primera aproximación permite pensar en las educaciones comparadas y ayuda a explicar ciertos procesos sociales y educacionales complejos como lo es la evaluación de los aprendizajes.

En segundo lugar, suponiendo que la lectura global crea un discurso universal para las distintas regiones, se utilizarán los aportes de Popkewitz (2005) para profundizar y dar cuenta de qué manera "aterriza" el concepto de evaluación formativa tanto en la CABA 
como en Finlandia; la noción de traveling libraries clarifica de qué manera se trasladan los supuestos alrededor del mundo.

Ahora bien, el proceso a llevarse a cabo tendrá en cuenta "hasta qué punto podemos aprender algo del valor práctico del estudio de sistemas educativos extranjeros" (Cowen, 2000, p. 13). Por ese motivo y dado que la educación está inserta en lo social, está contextualizada y relacionada con otros elementos, es que Cowen también nos ayudará a conocer de qué manera trabaja la noción de evaluación formativa en cada región de manera singular, en lo local.

Para abarcar todas las contribuciones propuestas al comienzo, se optará por complementar el discurso global de evaluación formativa que prima con el discurso local de cada una de las regiones escogidas. Entonces esta investigación será una herramienta que intente plantear que "el mundo es una mezcla de lo global y lo local" (Cowen, 2000, p. 20).

En una segunda instancia, utilizando la noción de traveling libraries (Popkewitz, 2005), se analizarán los documentos para ver de qué manera se reconstruyen y ensamblan los discursos de evaluación formativa en los distintos entornos culturales. Esta noción nos permite reconocer cómo las culturas locales se van adaptando y reorientando a las ideas del exterior en formas históricamente específicas. Las ideas educativas siempre han viajado por todo el mundo, sin embargo, hay que dar cuenta del proceso de recontextualización (Ball, 2002).

Por último, sabiendo que detrás de las estrategias de evaluación existe un objetivo por parte del docente que evalúa, se analizará la configuración del alumno al realizar las evaluaciones. ¿Cuál es el objetivo final de la evaluación? ¿Cómo tiene que responder el alumno? ¿Qué se espera del alumno?

A lo largo de todo el análisis de los documentos se intentará responder a estas cuestiones tomando los aportes de Hacking (2006), quien afirma que el estudio de ciertas ciencias crean tipos de personas que, en cierto sentido, no existían antes. Es decir, los distintos aterrizajes de la concepción de evaluación y las diferentes propuestas que se promueven por parte de los ministerios serán analizadas a luz del making-up people: esta noción proporciona una manera de hablar y de interpretar la evaluación de los aprendizajes y la manera en la que se pone en juego en el mundo. Este análisis se hará a su vez de la mano de Fenstermacher y Soltis (1998) que propondrán un marco teórico para encasillar el tipo de docentes que se presenta por parte de las distintas regiones.

\section{Análisis de los documentos: Finlandia y CABA}

\subsection{Contextualización: "La evaluación de los aprendizajes"}

La evaluación de los aprendizajes es una práctica social anclada en un contexto determinado: está conceptualizada e interpretada con significados distintos en las diferentes regiones; se hacen usos dispares, con fines e intenciones diversas, utilizando variedad de instrumentos, siguiendo principios y normas diferentes para dar a entender que, en su aplicación, sigue criterios de calidad.

Desde siempre, los sistemas de evaluación han perdurado en lo que conocemos como el sistema escolar. Lo interesante aquí es determinar de qué manera se inscribe un tipo de práctica -la evaluación formativa- en la contemporaneidad de nuestros tiempos, en el 
contexto actual; es decir, desarmar el concepto de "evaluación" para desnaturalizar su condición hegemónica ya que se ha vinculado a la evaluación con la acreditación en la mayoría de los institutos escolares de la ciudad de Buenos Aires. Más aún, resaltar la inserción de esta práctica en lo que se define como modernidad.

Siguiendo la línea de pensamiento de Foucault (1994), la evaluación formativa puede caracterizarse como una práctica moderna; un práctica que permite el desarrollo de una actitud particular. Siendo la modernidad un modo particular de relacionarse con y frente a lo que sucede cotidianamente, esta práctica provee al alumno una manera de pensar y de sentir diferente ya que incorpora el ejercicio de trabajar sobre sí mismo, en su pensamiento crítico y en su relación con el mundo real.

Por lo tanto, al evaluar formativamente el aprendizaje del alumno, se pasa del conocimiento tradicional basado en el contenido, a la incorporación de nuevos conocimientos que forman parte de una novedad: el sujeto como actor activo y reflexivo, configurando una nueva práctica de evaluación, de un nuevo sistema educativo. Además, para poder incorporar esta actitud de modernidad, el individuo necesita ser consciente de que es objeto de una elaboración ardua y compleja que no se detiene.

Esa conciencia la aprende a través de la evaluación formativa porque esta práctica genera life-long learners', genera la actitud -en el alumno- de querer aprender constantemente, recibiendo feedback a lo largo de todo el desarrollo educativo para poder inventarse y elaborarse a sí mismo una y otra vez. Por este motivo, la evaluación debería ser considerada como un proceso y no como un suceso, y construirse en un medio y nunca en un fin; la evaluación es el proceso a través del cual criticamos lo que somos, lo que tenemos, lo que alcanzamos pero también a través del cual experimentamos la posibilidad de conducir y modificar esas críticas que actúan como límites (Foucault, 1994).

Esta actitud moderna implicaría lo que para Kant (1784) es salirse del estado de tutela. En el contexto de la evaluación formativa, se le permite al alumno operar por él mismo sobre sí mismo, sabiendo que "la vocación de todo hombre es a pensar por sí mismo" (Kant, 1784, p. 8). Por tanto, esta práctica busca dejar a un lado la racionalidad técnica y el aprendizaje memorístico para dar lugar a la evaluación formativa y el aprendizaje significativo, dándole al alumno cierto grado de libertad y autonomía de manera tal que pueda conducirse en las circunstancias diarias de la vida.

Como parte de la modernidad, la evaluación formativa atiende a los alumnos de manera personal, intentando reestructurar su conocimiento a partir de las actividades que llevan a cabo e incitándolos en el libre pensar para llegar a convertirlos en algo más que una máquina que repite: los convierte en alumnos capaces de construir su propio pensamiento. Esto es un ejemplo de que el protagonista de la modernidad es el sujetoalumno; el foco está puesto fundamentalmente en los logros que él puede realizar por sí sólo y, más aún, la evaluación formativa evalúa la habilidad que tiene el alumno de poder inventarse a sí mismo y auto-regularse. La forma de regulación que ejerce la evaluación formativa supone intervenir necesariamente durante el período de tiempo dedicado a una unidad de formación, adaptando la enseñanza antes de que se califique al alumno o se decida su aprobación o desaprobación. 
Por último, cabe señalar que la práctica de evaluación formativa y significativa surge en el contexto de la modernidad por el carácter reflexivo que requiere el proceso. Como parte de esta actitud moderna, el alumno, a través de la evaluación formativa y significativa, consigue diferenciarse de las modalidades tradicionales manteniéndose en contacto con lo que hace y reflexionando sobre su propia acción (Giddens, 1990). Es decir, consigue definir su ethos, entendiéndolo como el conjunto de rasgos y modos de comportamiento que conforman el carácter o la identidad del alumno.

De esta manera, el desempeño escolar de los individuos es examinado constantemente por los alumnos y, con ayuda de las retroalimentaciones de los docentes, reformado a la luz de la nueva información que se obtiene a partir de las prácticas de evaluación. Por tanto, "la modernidad está totalmente constituida por la aplicación del conocimiento reflexivo" (Giddens, 1990, p. 47).

Todo proceso de evaluación pone en evidencia múltiples aspectos, relacionados con las características y procesos de la institución educativa, los proyectos institucionales, los estilos de gestión, las propuestas curriculares y editoriales, las particularidades de los docentes y de los alumnos. La multidimensionalidad de los actos evaluativos, producto de sus articulaciones y sobre determinaciones, conduce a poner en duda la unidad y coherencia de lo que se designa con el término "evaluación".

Acorde a los documentos elegidos, es menester resaltar la noción de evaluación que se presenta formalmente desde los distintos ministerios. Por un lado, en Finlandia, la metodología de evaluación ha abandonado las memorizaciones del sistema educativo y hace énfasis en el desarrollo de la curiosidad, la creatividad y la experimentación: no es una cuestión de transmitir información, sino que es más importante aprender a pensar (Finnish National Board of Education, 2003).

Por otro lado, el Gobierno de la CABA plantea que se evalúa de manera tal de construir un mapa del estado actual del progreso de los aprendizajes de los estudiantes para poder planificar de acuerdo al logro de los objetivos. Este tipo de evaluación permite que el docente cuente con la información necesaria acerca del progreso significativo de sus estudiantes (Ministerio de Educación de la Ciudad de Buenos Aires, 2013).

A continuación, se hará un análisis exhaustivo de los documentos a ser trabajados en función de distintas nociones que atravesarán y moldearán el artículo: la noción de "leer lo global" propuesta por Cowen (2000), la noción de "traveling libraries" (Popkewitz, 2005) y la noción de "making-up people" (Hacking, 2006).

\subsection{Cowen: "leer lo global"}

Un primer punto a señalar para desarrollar el análisis de una manera adecuada es la necesidad de "leer lo global" (Cowen, 2000). El proceso de conocer los discursos universales que perduran hoy en día en relación a la evaluación de los aprendizajes es indispensable para luego observar las dinámicas educacionales de cada región.

Un estudio comparativo que refiere a una temática particular "se concentra alrededor de estas ideas unificadoras y los académicos que leen lo global en diferentes momentos piensan en diferentes versiones de esto” (Cowen, 2005, p. 16). Por lo tanto, ¿¿cuáles son esos discursos externos, universales, globalizados, que juegan como punto de referencia en el campo de la evaluación de los aprendizajes? ¿Qué se dice sobre la evaluación de los aprendizajes? 
Si bien al mirar en profundidad y analíticamente los documentos tiene especial preponderancia la evaluación formativa, se admite un discurso más amplio que se extiende por lo que plantean los documentos locales. Philippe Perrenoud (2010) afirma que "las pedagogías diferenciadas están ya a la orden del día y la evaluación formativa ya no es una quimera, pues ha dado lugar a numerosos ensayos en diversos sistemas" (p. 15). Es decir, parecería ser que la evaluación formativa, en la contemporaneidad de nuestros tiempos, permanece como "lo global".

Esto se debe a que, por ejemplo, Boud y Falchikov (2005) plantean que "aunque la función formativa es a menudo eclipsada a diario por el predominio de las evaluaciones sumativas, se ha producido una renovación de intereses en la evaluación formativa en los últimos años" ${ }^{1}$ (p. 35). Y más aún, diversos investigadores como Black y Wiliam (1998) o Elwood (2007) identifican en la evaluación formativa, lo que ellos denominan como "buenas prácticas".

Entonces, considerando que el discurso de la evaluación formativa aparece en los documentos a analizar y se repite este patrón en la literatura contemporánea sobre evaluación, podríamos constatar que la evaluación formativa permanece como "lo global", como lo que circula hoy en el mundo a través de las distintas regiones. Tanto los documentos como los textos utilizados definen, en mayor o menor medida, a la evaluación formativa como una actividad sistemática y continua que proporciona información necesaria sobre el proceso educativo y que orienta a los estudiantes a través de la retroalimentación.

El desafío está en la "reconstrucción en estos procesos sociales que suponen la destrucción del pasado y la redefinición del futuro" (Cowen, 2000, p. 35); es decir, este nuevo discurso admite la posibilidad de dejar a un lado las evaluaciones tradicionales a favor de la selección y construir evaluaciones modernas a favor del aprendizaje (Perrenoud, 2010).

A través de la lectura de lo global se puede identificar que los argumentos en torno a la evaluación en las diferentes regiones surgen gracias a la interdependencia mundial: acorde a cómo evolucione el mundo, acorde a las cuestiones que se presentan, acorde a las diferentes estrategias de evaluación que existan y perduren, acorde a los resultados académicos de las naciones, es que las concepciones locales en torno a la evaluación cambiarán o permanecerán para poder lograr mantener una educación actualizada y significativa en la formación integral del estudiante. En consecuencia, necesitamos de la lectura de lo global para comparar las tendencias de evaluación de ambas regiones analizadas y verificar la presencia o ausencia de este código educativo que predomina en el mundo actual.

Este nuevo patrón educativo sobre el que se trabaja -la evaluación formativa- puede percibirse de maneras diversas en los documentos con los que se busca trabajar. Por un lado, en el caso de Finlandia, a la hora de definir el currículum que va a regir para la escuela secundaria, se expone la necesidad de garantizarle a los estudiantes la oportunidad de completar sus estudios de manera flexible en función de su progreso y del feedback que le den sus guías académicos o docentes. Es decir, por detrás de los objetivos se esconde el propósito de que los alumnos tomen sus propias decisiones, si

\footnotetext{
${ }^{1}$ Traducción realizada por la misma autora. El texto original está en inglés.
} 
dejar de aprovechar los aportes constructivos que le ofrezcan los docentes (Finnish National Board of Education, 2003)2.

Los argumentos globales de la evaluación formativa están presentes en el documento de Finlandia porque se percibe al aprendizaje como el proceso a través del cual los estudiantes interpretan la información recibida en interacción con otros alumnos, con los profesores y con el entorno y en base a sus conocimientos previos ya incorporados (esta es la noción de aprendizaje significativo). A pesar de que se promueva el trabajo independiente, los alumnos tienen que ser guiados para ser conscientes de su propio proceso de enseñanza-aprendizaje.

La educación en Finlandia tiene en cuenta el hecho de que las habilidades de los estudiantes varían y por eso necesitan de los docentes para que supervisen sus estudios de maneras diferentes. La presencia de cierto grado de heterogeneidad nos permite afirmar que Finlandia hace hincapié en los procesos individuales de cada alumno, teniendo en cuenta la importancia de los procesos formativos para crear, a su vez, "lifelong learning and continuous self-development" (Finnish National Board of Education, 2003, p. 12).

En conclusión, desde el Ministerio de Educación de Finlandia, se promueve el discurso global de evaluación formativa ya que definen a la evaluación como el proceso a través del cual se promueve la auto-evaluación en complemento con el feedback formativo para identificar el progreso y los resultados de aprendizaje: "the role of assessment is to provide students with feedback on how well they have met the objectives of the course and on their progress in that subject" (Finnish National Board of Education, 2003, p. 224).

Por otro lado, desde el Ministerio de Educación de la CABA, también pueden identificarse argumentos que se inscriben en el paradigma de la evaluación formativa sobre todo por la insistencia en generar aprendizaje significativo. Además, al igual que en Finlandia, se propone que el sistema educativo adquiera la flexibilidad suficiente como para asumir escenarios cambiantes y poder brindarle a los estudiantes la habilidad de aprender durante toda la vida. Las nociones de life-long learnering y de aprendizaje significativo son indispensables en el marco de la evaluación formativa.

El sistema educativo de la CABA admite entonces que la evaluación debe ser "formativa, formadora y sumativa" (Ministerio de Educación de la Ciudad Autónoma de Buenos Aires, 2013, p. 61) de manera tal de poder comprender los procesos y los resultados para mejorar los procesos de aprendizaje de los alumnos. Es decir, en el documento formal -el Diseño Curricular- se presenta el discurso global de evaluación formativa ya que se promueve el deseo de proporcionar una mirada integral y de mejorar los procesos de enseñanza a través de la devolución fundamentada de docentes y de los propios compañeros de curso (p. 145). También, como parte del discurso de la formatividad, se explica cómo este tipo de evaluación permite conocer lo que el estudiante sabe hacer y qué es lo que le falta aprender, es decir, conocer su progreso y motivarse con sus logros.

Si bien desde la Ciudad de Buenos Aires el discurso no se orienta, como en Finlandia, a la individualización de las trayectorias escolares, se admiten procesos heterogéneos

2 Cita textual: "flexible arrangements for progression of studies and provision of special support as required. The curriculum must be drawn up so as to provide students with an opportunity to make individual choices" (2003, p. 8). 
orientados al seguimiento de los aprendizajes de los estudiantes con el objetivo de perfeccionar las trayectorias, "brindando una mayor autonomía y responsabilidad a los estudiantes” (Ministerio de Educación de la Ciudad Autónoma de Buenos Aires, 2013, p. 146). De esta manera, el trabajo se orienta, en el marco de la evaluación formativa, a trabajar con los errores de los alumnos de manera constructiva para generar aprendizaje significativo.

\subsection{Popkewitz: “Traveling libraries”}

¿Cómo se realiza la traducción de este discurso global? “ ¿Cómo se realiza la traducción de aquello que es externo al colegio?” (...) “¿Cómo pasa de ser una idea externa a ser una práctica educativa?” (Cowen, 2005, p. 17).

Para que la evaluación formativa se ponga en juego, no es suficiente conocer de qué se trata y plantearlo como un paradigma teórico a tener en cuenta. La evaluación formativa se pone en juego en el momento en el que ocurre la traducción, en el momento en el que se conjugan elementos externos e internos.

Para combinar el discurso teórico con lo que sucede en el interior de los sistemas educativos escogidos, es necesario tener en cuenta el concepto de "traveling libraries" (Popkewitz, 2005). Este autor demuestra que el viaje de los discursos no es desde un centro a la periferia ni de un punto fijo a otro punto fijo, sino más bien, está mediado por los marcos discursivos socio-históricos y específicos que redefinen el interés de cada nación. Esto permite comprender que la tendencia visible y popular de la evaluación formativa que se traslada actualmente alrededor del mundo, es reinterpretada y redefinida por cada región a su manera.

La noción de "travelling libraries" da especial importancia al entramado de redes a través del cual se da inteligibilidad a los cambios y proporciona una manera de pensar acerca de lo que aparece en la superficie de los debates educativos contemporáneos. Es decir, este concepto proporciona una manera de considerar la globalización y la localización como procesos relacionados y superpuestos de manera diferente en los distintos lugares.

En función de estos aportes y de lo presentado como evaluación formativa desde los diferentes documentos, podemos analizar las dimensiones socio-históricas que delimitan el campo de la evaluación en los sistemas educativos. Es decir, la manera en la que "aterriza" el discurso de la evaluación en las distintas regiones estará influenciado por los intereses que tenga cada nación.

Por un lado, en el caso de la Ciudad de Buenos Aires, a lo largo de los años, la escuela media se ha enfrentado a numerosos cambios en el campo pedagógico que hacen que, en la actualidad, tenga un significado distinto al que tenía en el momento fundacional de los sistemas educativos occidentales. Tras la promulgación de la Ley Nacional de Educación 26.206 del 2006, el nivel medio o secundario se ha convertido en el nuevo piso de la escolaridad obligatoria (Tenti, 2000, p. 2). Esta nueva disposición afecta notablemente los modos de hacer las cosas en el interior de las instituciones, dando lugar a una serie de transformaciones en los dispositivos y procesos escolares.

El enfrentamiento a una nueva disposición de los contenidos escolares, de las estrategias de enseñanza, de los actores participantes dentro del sistema, exige a los alumnos incorporar ciertas habilidades que se correspondan con los lineamientos del siglo XXI: "necesidad de acercar la situación de la escuela secundaria a la realidad 
compleja del siglo XXI, cuya sociedad es más flexible y cambiante" (Ministerio de Educación de la Ciudad Autónoma de Buenos Aires, 2013, p. 73).

La obligatoriedad de la escuela secundaria, entonces, introduce la necesidad de transformar el proceso de enseñanza-aprendizaje, buscando promover que el aprendizaje en este nivel sea significativo de manera tal que se construyan alumnos autónomos y capaces de razonar de manera personal:

El Ministerio de Educación de la Ciudad Autónoma de Buenos Aires ha puesto el eje de toda política educativa en el aprendizaje significativo de los estudiantes. Se ha iniciado un proceso de llevar el sistema educativo orientado en el docente a un sistema orientado en el aprendizaje del alumno y la nueva organización institucional necesaria para que el aprendizaje suceda. (Ministerio de Educación de la Ciudad Autónoma de Buenos Aires, 2013, p. 25)

Para promover y contribuir al aprendizaje significativo, resulta necesario hacer hincapié en el campo de la evaluación ya que, para elevar la calidad de los aprendizajes, el rol de la evaluación educativa es central: es un componente esencial del proceso que transcurre en la escuela.

En la contemporaneidad de nuestros tiempos, la evaluación se presenta como una problemática educativa que, a nivel micro, forma parte del debate didáctico y constituye uno de los problemas centrales para los docentes de la escuela media. Con el objetivo de transformar la evaluación tradicional, los docentes intentan visibilizar las adquisiciones y los modos de razonar de cada alumno haciendo de la enseñanza, una más personalizada.

La evaluación está en proceso de dejar a un lado su función selectiva y aumentar una función pedagógica. A pesar de que todos los tipos de evaluación sean indispensables para la mejora en la calidad de los aprendizajes, se reconoce a la evaluación formativa como la práctica crucial para generar aprendizaje significativo: "la evaluación de carácter formativo permite al estudiante conocer qué es aquello que sabe hacer y qué es lo que le falta aprender $(\ldots)$ debe estar orientada al seguimiento de los aprendizajes de los estudiantes con el objetivo de perfeccionar las trayectorias escolares" (Ministerio de Educación de la Ciudad Autónoma de Buenos Aires, 2013, p. 146).

Sin embargo, tanto los docentes como los directivos se enfrentan a diario con dificultades que se presentan a la hora de definir los criterios de evaluación. Frente a las adversidades de la actual escuela secundaria argentina, este tipo de evaluación está lejos de ser puesta en práctica en todas las instituciones con coherencia, ya que lucha contra los numerosos obstáculos institucionales: el número de alumnos, la sobrecarga de los programas, la concepción de los medios de enseñanza y las didácticas.

Por otro lado, tomando los aportes de Simola (2005), podemos comprender el marco socio-histórico en el cual se inscribe la evaluación formativa en Finlandia. En las últimas décadas, Finlandia ha desarrollado políticas educativas que lo llevaron en pos del éxito y del reconocimiento internacional.

El alto rendimiento del sistema finlandés parece ser atribuible a toda una red de factores relacionados entre sí: las actividades de interés y de ocio, las oportunidades de 
aprendizaje proporcionadas por las escuelas, el apoyo de los padres y la participación ${ }^{3}$, el contexto social y cultural del aprendizaje y de los estudiantes de todo el sistema educativo. Pero la historia de éxito de Finlandia es sumamente reciente.

El sistema de enseñanza secundaria se desarrolló sólo en la década de los 70’, pero al mismo tiempo se llevó a cabo muy rápidamente y de forma sistemática, incluso de una manera bastante totalitaria. La enseñanza y el aprendizaje siempre fueron muy tradicionales e implicaron la exposición por parte del docente en frente de todo el grupo de alumnos (Simola, 2005). A pesar de que la cultura finlandesa aún tenga incorporado un elemento significativo de mentalidad autoritaria, conservadora y obediente, hoy en día las cosas han llegado a ser más independientes y, en consecuencia, la evaluación sigue una pedagogía más independiente: "each student will draw up his or her own individual study plan" (Finnish National Board of Education, 2003, p.8) y, además, "assessment will encourage students in a positive way to set their own objectives and to readjust their working methods" (p. 224).

La cultura arcaica prevalece pero hay una presencia de confianza social y de apreciación a los maestros que cumplen su labor de manera comprometida. En la actualidad, las dinámicas en el aula están organizadas en función de trabajo individual o grupal de los alumnos mientras que el docente los guía dándole feedback al trabajo que van realizando. Por lo tanto, en este nuevo contexto donde se pone en juego no sólo la interacción docente-alumno sino también la de alumno-alumno, la evaluación formativa toma un rol indispensable.

La evaluación alentará a los estudiantes de una manera positiva para establecer sus propios objetivos y reajustar sus métodos de trabajo. A su vez, proporcionará a los estudiantes con información sobre lo bien que se han cumplido los objetivos del curso y sobre los progresos realizados en cada una de las materias.

A diferencia de la CABA, en Finlandia la evaluación se inscribe en un contexto de confianza, donde el control y la regulación tiene un rol poco observable. Esto se debe más que nada al desarrollo de las políticas educativas que se dieron a partir de 1995. Desde principios de los 90', el discurso educativo estatal se ha centrado en la evaluación como la herramienta fundamental para un desarrollo de calidad. Mientras que anteriormente se había creído que las metas educativas podían alcanzarse ajustándose a normas estrictas, la convicción en los 90' fue que su consecución requería el establecimiento de objetivos nacionales básicos y la evaluación de los logros a la luz de los posteriores resultados.

En conclusión: "the Finnish 'secret' of top-ranking may therefore be seen as the curious contingency of traditional and post-traditional tendencies in the context of the modern welfare state and its comprehensive schooling" (Simola, 2005, p. 466).

\subsection{Hacking: "making-up people"}

Ahora bien, la interacción entre los discursos cosmopolitas democráticos y las reformas educativas actuales o contextos locales (Friedrich, 2014) admite un proceso de traducción y recontextualización de lo que se conoce como evaluación formativa (Ball, 2002). Fue de especial relevancia atender a las particularidades locales y a la

\footnotetext{
3 'Students' parents or guardians must be provided with sufficient opportunities to familiarise themselves with the work of the upper secondary school. Co-operation with parents will support the prerequisites for study" (Finnish National Board of Education, 2003, p. 18).
} 
formulación y promulgación de políticas para identificar cuáles son las similitudes o diferencias con los patrones generales y visibilizar la manera en la que se pone en juego la noción de "traveling libraries".

No obstante, sabiendo que por detrás de cualquier acto evaluativo existe un objetivo por parte del docente que lleva a cabo la acción, queda por explorar el tipo de sujeto con el que se trabaja en las distintas regiones. Como respaldo teórico, tomaremos la noción de "making-up people" (Hacking, 2006) para enriquecer el argumento de que, con la evaluación formativa, se construyen nuevos participantes antes inexistentes.

Por un lado, en lo que respecta al Diseño Curricular de Finlandia, se ha destacado la presencia indispensable de la confianza y la libertad. La evaluación en esta región no sólo admite ciertas ideas y características de la evaluación formativa, sino que presenta un alumno libre y capaz de regular sus propios aprendizajes de manera autónoma, con la seguridad de tener una guía constructiva que lo ayude a alcanzar sus objetivos de aprendizaje: "students are seen to be the constructors of their own learning, competence and views of the world" (Finnish National Board of Education, 2003, p.12).

En consecuencia, el docente tiene una postura que, en palabras de Fenstermacher y Soltis (1998), podría denominarse "terapeuta": un docente cuyo énfasis está puesto en el alumno y en lo que él puede lograr, en su desarrollo psíquico e intelectual para escoger la ruta que más potencie el aprendizaje del estudiante: "counselling and guidance from an integrated whole, which aims to support students at different stages of their upper secondary school studies and to develop their capabilities to make choices and find solutions concerning education, training and future career" (Finnish National Board of Education, 2003, p.18).

Por otro lado, en el caso de la CABA, el docente se asocia al paradigma del "ejecutivo" (Fenstermacher y Soltis, 1998): un docente que planifica, que recibe instrucciones, que lo orientan a la hora de poner en juego ciertas estrategias y cuyo rol es fundamental para que la clase tenga sentido y ocurra. Existe un documento de carácter orientativo para los docentes que "propone un desarrollo más exhaustivo que incluye los componentes del material anterior -propósitos y contenidos- junto con la presentación de los objetivos de aprendizaje por año de estudio y alcances y comentarios para el desarrollo de los contenidos, incluyendo sugerencias para su enseñanza" (Ministerio de Educación de la Ciudad Autónoma de Buenos Aires, 2013, p. 36).

Por su parte, el alumno posee menos libertad a la hora de elegir sus propios cursos para completar la escolaridad secundaria obligatoria y, además, está sumamente influenciado por las aptitudes que, desde el Ministerio, esperan que tenga el futuro egresado de la NES: "metas que todos los egresados del nivel secundario DEBEN alcanzar. Dichas aptitudes pueden definirse como los recursos cognitivos y comportamentales que debería adquirir el alumno en el trayecto de su educación media para enfrentar las demandas de los estudios superiores" (Ministerio de Educación de la Ciudad Autónoma de Buenos Aires, 20013, p. 74).

En resumen, si vivimos en un mundo en donde los objetivos se mueven constantemente, la interacción entre un docente y un alumno -por ejemplo- se va a ver modificada. Y ya que cambian las interacciones y los objetivos de la evaluación, las personas ya no son exactamente la misma clase de gente que eran antes.

A pesar de Finlandia y Buenos Aires tener objetivos distintos en cuanto a la educación, las estrategias de enseñanza y los aspectos más delicados de la evaluación, en los 
documentos ambos dos poseen un capítulo específico que refiere a la evaluación y otro capítulo que refiere a las habilidades o aptitudes a lograr por parte del docente y el alumno. Esa similitud podría considerarse como un aspecto que hoy está globalizado en los diseños curriculares alrededor del mundo.

\section{Conclusión}

A lo largo de este trabajo académico se ha intentado exponer, describir y explicar las distintas concepciones construidas en torno a la temática de la evaluación. Siguiendo los argumentos propuestos por ciertos investigadores de educación comparada, se ha hecho un estudio comparativo entre Finlandia y la Ciudad Autónoma de Buenos Aires, que permite visibilizar los modos y las configuraciones que se inscriben en la noción de evaluación formativa de cada región.

La propuesta de este artículo ha sido complejizar las ideas formales de evaluación que se presentan en los Diseños Curriculares de las distintas regiones. De tal manera, se intentó percibir cómo "aterrizan" las características de la evaluación formativa en Finlandia y Buenos Aires, cómo se traslada el concepto de evaluación formativa al campo formal de los Diseños Curriculares.

El objetivo primordial fue identificar cómo se leen las propuestas o los argumentos de evaluación, sabiendo que los docentes que están al servicio de la educación y la escuela poseen sus propias estrategias de enseñanza y cargan con un tinte de subjetividad. Es decir, explicar cómo se configuran los enfoques, cuáles son las similitudes y diferencias entre las regiones y qué orientaciones se da a los profesores a la hora de poner en juego la evaluación.

Una primera aproximación refleja que la evaluación existe desde que se creó el dispositivo escuela y, en tanto estrategia global, se hacen distintas lecturas de la misma en las diferentes regiones analizadas. De todas maneras, ambos inscriben la noción de evaluación formativa en sus estrategias de evaluación de los aprendizajes y hacen hincapié en la individualización de los procesos escolares acorde a las capacidades de los alumnos. Además, intentan construir individuos que aprendan a lo largo de toda la vida, es decir, life-long learners.

También ambos explican el deseo de que los alumnos sean individuos autónomos, capaces de incorporarse al mundo laboral sin dificultades sino, más bien, con las capacidades suficientes como para enfrentar lo cotidiano de la vida real. Sin descartar que las tareas escolares están en constante evolución -por los avances tecnológicos y las mejores en el campo pedagógico- el dispositivo de evaluación sigue existiendo en todas las escuelas a lo largo del mundo, de alguna u otra manera.

Ahora bien, ¿qué se puede rescatar de Finlandia que no se haya puesto en juego aún en la Ciudad de Buenos Aires? El argumento de la evaluación formativa ya no es algo para o de algunos pocos sino que está, cada vez más, girando alrededor del mundo. La diferencia reside, como fue explicado anteriormente, en el grado de utilización de las características o en la manera en la que la ponen en juego tanto los docentes como los alumnos.

Entonces, afirmando que la evaluación formativa aumenta la calidad de los aprendizajes, la CABA debería formar a los docentes de manera más terapéutica y no tan ejecutiva. Es así como el docente incorporará el ejercicio de reflexionar sobre el 
aprendizaje de sus alumnos -como actitud moderna- para luego guiarlos en la construcción de su autonomía.

\section{Referencias}

Acevedo, P. A. (2001). La evaluación en una concepción de aprendizaje significativo. Valparaíso: Ediciones Universitarias de Valparaíso - Pontificia Universidad Católica de Valparaíso.

Apel, J. (1998). Evaluar e informar en el proceso de enseñanza-aprendizaje. Buenos Aires: Aique Grupo Editor.

Ball, S. (2002). Grandes políticas, un mundo pequeño. Introducción a una perspectiva internacional en políticas educativas. En M. Narodowski, M. Nores, y M. Andrada (Coord.), Nuevas tendencias en Políticas Educativas (pp. 103- 129). Buenos Aires. Ediciones Juan Granica.

Black, P. y Wiliam, D. (1998). Inside the black box: Raising standards through classroom assessment. Londres: Kings College.

Boud, D. y Falchikov, N. (2005). Redesigning assessment for learning beyond higher education. Higher Education Research and Development Society of Australasia, 28, 34-41.

Cowen, R. (2005). Los códigos secretos de los sistemas educativos: Leyendo las "Piedras Roseta”. Boletín de la Academia Nacional de Educación, 63, 12-20.

Elwood, J. (2007). Formative assessment: Possibilities, boundaries and limitations. Assessment in Education: Principles, Policy \& Practice, 13(2), 215-232. doi:10.1080/09695940600708653.

Finnish National Board of Education. (2003). National Core Curriculum for Upper Secondary Schools. Helsinki: Studio Viiva Oy.

Fenstermacher, G. y Soltis, J. (2001). Enfoques de la enseñanza. Buenos Aires: Amorrortu.

Friedrich, D. (2014). Global microlending in education reform: Enseñá por Argentina and the neoliberalization of the grassroots. Comparative Education Review, 58(2), 296-321. doi:10.1086/675412

Foucault, M. (1984). Qu'est-ce que les Lumières? En J. Dávila (Ed.), ¿Qué es la Ilustración? (pp. 19-46). Madrid: Actual.

Giddens, A. (1990). Consecuencias de la modernidad. Madrid: Alianza Editorial.

Hacking, I. (2006). Making up people. London Review of Books, 28(16), 23-26.

Kant, I. (1784). Respuesta a la pregunta: ¿qué es la Ilustración? Revista Colombiana de Psicología, 14, 7-10.

Meyer, J. y Ramírez, F. (2000). La institucionalización mundial de la educación. En J. Schriewer (Comp.), Formación del discurso en la educación comparada (pp. 91-111). Barcelona: Pomares.

Ministerio de Educación de la Ciudad Autónoma de Buenos Aires. (2013). Nueva Escuela Secundaria de la Ciudad de Buenos Aires. Diseño Curricular. Ciclo Básico 2014-2020. Recuperado de http://www.buenosaires.gob.ar/areas/educacion/recursos/NESCB2014_web.pdf2013

Perrenoud, P. (2008). La evaluación de los alumnos. De la producción de la excelencia a la regulación de los aprendizajes. Buenos Aires: Colihue.

Pineau, P., Dussel, I. y Carusso, M. (2001). La escuela como máquina de educar. Tres escritos sobre un proyecto de la modernidad. Buenos Aires: Paidós. 
Popkewitz, T. (2013). Pisa, números, estandarización de la conducta y la alquimia de las materias escolares. Profesorado, 17(2), 47-64.

Scriven, M. (1967). The methodology of evaluation. En Tyler, R. W., Gagné, R. M. y Scriven, M. (Eds.), Perspectives of curriculum evaluation, 39-83. Chicago, IL: Rand McNally.

Simola, H. (2005). The Finnish miracle of PISA: Historical and sociological remarks on teaching and teacher education. Comparative Education Review, 41(4), 455-470. doi:10.1080/03050060500317810

Tedesco, J. C. y López, N. (2002). Desafíos a la educación secundaria en América Latina. Revista CEPAL, 76, 55-69.

Tenti, E. (2000). Culturas juveniles y cultura escolar. Buenos Aires: IIPE-UNESCO. 